\title{
CONSTRUCTING ISOTOPIES ON NONCOMPACT 3-MANIFOLDS $\left({ }^{1}\right)$
}

\author{
BY
}

\author{
MARIANNE S. BROWN(2)
}

\begin{abstract}
We consider the question "When are two homeomorphisms of a noncompact 3-manifold onto itself isotopic?" Roughly, the answer is when they are homotopic to each other. More precisely, this paper deals with the question for irreducible 3-manifolds which either have an infinite hierarchy or have such a hierarchy after the removal of a compact set. Manifolds having the first property are called end-irreducible; the others are called eventually endirreducible. There are two results for each type of manifold depending on whether the homotopy between the two homeomorphisms sends the boundary of the manifold into itself or not.
\end{abstract}

0 . Introduction and acknowledgements. We consider here the problem of when two homeomorphisms of a noncompact 3-manifold are isotopic. Waldhausen [13] has answered this question for compact, irreducible 3-manifolds which admit a hierarchy-roughly the answer is-when they are homotopic. We prove noncompact versions for manifolds which either have an "infinite hierarchy," or which have such a hierarchy after removing a compact subset. For the first class it will not be necessary to assume the homotopy is proper. For the second class of manifolds, in $\$ 3$ we show it is essential that the homotopy respect the fact that the manifold has a hierarchy only far out, i.e., it must be proper.

The proofs of these theorems are quite long. $\$ \$ 1$ and 2 and the main lemma in $\$ 3$ prove lemmas that are used in all of the results. $\$ 3$ contains the results in the eventually end-irreducible case and $\$ 4$ contains the results for end-irreducible manifolds. The reader might find it helpful to read a sketch of the proof in [18]. Also the author has a preprint version with a fuller exposition.

I should like to express my gratitude to my husband, Edward M. Brown, for all the help he has given me. It is impossible to try to list all the ways in which he helped, but at least let me thank him for suggesting the problem, giving me unfailing encouragement and support, and for innumerable helpful conversations. I should like to thank Dennis Sullivan for being my thesis advisor with all that it entails, and David Stone for having several very helpful conversations with me.

Received by the editors November 2, 1971 and, in revised form, June 25, 1972.

AMS (MOS) subject classifications (1970). Primary 58D05; Secondary 55A99, 57C99.

(1) This paper contains the results of the author's thesis at the Massachusetts Institute of Technology under Dennis P. Sullivan.

(2) Supported by National Science Foundation grant GP-29076. 
Lastly, I recognize my debt to the Radcliffe Institute for its financial support and encouragement during the last two years and to the woman's liberation movement for having motivated me to think deeply about what I wanted from life. It is to my sisters in the woman's liberation struggle and to their allies (my husband included) that this paper is dedicated.

1. Preliminaries. We adopt the following conventions regarding terminology. By a manifold we will mean an orientable triangulable 3-manifold M. A surface in $\partial M$ is just some subsurface of $\partial M$. The results of Bing [3] and Moise [12] show that triangulability assumptions are not a serious restriction. Unless stated otherwise our surfaces and manifolds are connected. Also, unless stated to the contrary, a surface $F$ in a manifold $M$ is properly embedded, i.e., $F \cap \partial M \subset \partial F$. If $X$ is a finite polyhedron in a manifold $M$ (surface $F$ ) we let $U(X)$ denote a regular neighborhood of $X$ in $M$ (in $F$ ) in the sense of J. H. C. Whitehead [15]. Moreover, in general, choose a triangulation in which all subspaces, previously mentioned in an argument, are subcomplexes; construct its second derived, and take the closed star of the object in question. In the presence of a product structure, we will sometimes require that $U(\ldots)$ is in some sense compatible with the product structure. This will be indicated in the text. We use $\operatorname{cl}(X)$ to denote the closure of $X$ in $Y$, where $X$ is any subspace of a space $Y$. I will denote the unit interval.

If $X$ is a space, an isotopy of $X$ is a homotopy $J: X \times I \rightarrow X$ such that $J_{t}: X \rightarrow X$ defined by $J_{t}(x)=J(x, t)$ is a homeomorphism for each $t$ in $I$. When we say "Let $J$ be an isotopy of a bomeomorphism $b . .$. ", we will mean that $J_{0}=b$. A homotopy $H: X \times I \rightarrow X$ is rel a subspace $Y$ of $X$ if $H(y, t)=H\left(y, t^{\prime}\right)$ for $t$ and $t^{\prime}$ in $I$ and $y$ in $Y$. The same thing is meant by the phrases fixed on $Y$ and constant on $Y$.

Let $F$ be a surface (properly embedded) in a manifold $M$. If we cut $M$ along $F$, we obtain a manifold $\widetilde{M}$. ( $M$ is not necessarily connected.) We will also refer to $\widetilde{M}$ as the space resulting from splitting $M$ along $F$. $\widetilde{M}$ has two copies $F_{1}$ and $F_{2}$, of $F$ on its boundary. Denote by $p: \widetilde{M} \rightarrow M$ the map which identifies $F_{1}$ and $F_{2}$.

Let $F$ be a surface in a manifold $M$. Since $F$ and $M$ are orientable, $F$ disconnects any regular neighborhood $U(F)$. A homeomorphism $b$ of $M$ such that $h(F)=F$ interchanges the sides of $F$ if it maps points close to $F$ in one component of $U(F)-F$ into points in the other component.

As mentioned in the introduction the isotopy results are proved for irreducible manifolds. 'Such manifolds can be dealt with nicely by using incompressible surfaces. We now define these terms and state some properties (without proof) that will be constantly used. We follow Waldhausen [13] for the definitions and 
properties, but extend them to include noncompact surfaces and manifolds.

A system of surfaces in $M$ consists of finitely many pairwise disjoint surfaces in $M$. Let $F$ be a system of compact surfaces in $M . F$ is incompressible in $M$ if both of the following conditions hold.

(1) If $\Delta$ is a disc $M$ with $\Delta \cap F=\partial \Delta$, then $\partial \Delta$ bounds a disc in $F$.

(2) There does not exist a ball $B$ in $M$ such that $B \cap F=\partial B$.

It is known that a system of compact surfaces is incompressible in $M$ if and only if each component is incompressible in $M$. Also, if $F$ is a surface in $M$ different from the 2-sphere, $F$ is incompressible in $M$ if and only if $\operatorname{ker}\left(\pi_{1}(F) \rightarrow \pi_{1}(M)\right)=0$. If $F$ is a boundary component of a manifold $M, F$ is incompres. sible if in addition to (1) and (2), $F$ is not a plane. $M$ is boundary-irreducible if $\partial M$ is incompressible. A manifold $M$ is irreducible if every 2 -sphere bounds a ball in $M$.

Let $M$ be a manifold and $F$ a system of incompressible compact surfaces in $M$. Let $U(F)$ be a regular neighborhood of $F$. Then

(a) $\mathrm{cl}(M-U(F))$ is irreducible if and only if $M$ is.

(b) $\operatorname{ker}\left(\pi_{1}\left(M^{\prime}\right) \rightarrow \pi_{1}(M)\right)=0$ for each component $M^{\prime}$ of $\mathrm{cl}(M-U(F))$.

Suppose $M$ is an irreducible manifold which is either compact with nonempty boundary or noncompact. Then $M$ is aspherical, i.e., $\pi_{j}(M)=0$ for $j \geq 2$.

We will also need the notion of parallel surfaces. First, suppose $F$ and $G$ are compact surfaces in $M$. By an ambient isotopy of $M$ carrying $F$ onto $G$, we mean an isotopy $J: M \times I \rightarrow M$ of the identity to a homeomorphism $J_{1}$ such that $J_{1}(F)=G$. Now suppose $F$ and $G$ are compact surfaces in $M$ or $\partial M$. $F$ is parallel to $G$ if and only if there is an embedding $e: F \times I \rightarrow M$ such that $F=$ $e(F \times 0)$ and $G=e(\operatorname{cl}(\partial(F \times l)-F \times 0))$. Notice that if $F$ is parallel to $G$, then $G$ is parallel to $F$. Also, if $F$ and $G$ are parallel and both are in $M$, then they are isotopic by an isotopy which is constant on $\partial M$.

The first proposition permits us to isotope the homeomorphism on new surfaces of a hierarchy without changing the map where it has already been isotoped to the identity.

Proposition 1.1. Let $M$ be an irreducible manifold. Let $C$ be a compact irreducible manifold contained in $M$ and suppose $F$ is a system of compact in. compressible surfaces in $C$. Let $G$ be a closed incompressible surface in $C$ so that $G \cap F=\varnothing$.

Further, let $b$ be a homeomorphism of $M$, sucb that

(1) $b F_{i}=F_{i}$ and $b$ does not interchange the sides of $F_{i}$.

(2) $b$ is bomotopic to the identity by a bomotopy $H$ such that $H(G \times I) \sqsubset C$.

(3) $b(G)$ is incompressible in $C$.

Then there is an ambient isotopy of $C$ which is fixed on $F \cup \partial C$ carrying $b(G)$ onto $G$. 
Proof. This proof is quite similar to one by Waldhausen [13, Corollary 5.5]. Put $b(G)$ in general position with respect to $G$ by an isotopy which is fixed on $F \cup \partial C$, and change $b$ and $H$ according to this isotopy. Now $G$ intersects $b(G)$ transversally and $G \cap b(G)$ consists of pairwise disjoint simple closed curves.

The rest of the proof exhibits a set of "nice" isotopies of $C$ which first move $b(G)$ completely off $G$. We will show at that point that $b(G)$ is in fact parallel to $G$ and our conclusion follows.

We apply Proposition 5.4 in [13] to obtain "pieces," $\widetilde{G}$ and $\widetilde{b G}$, of $G$ and $b(G)$ respectively, and an embedding $f: \widetilde{G} \times I \rightarrow C$ such that $f(\widetilde{G} \times 0)=\widetilde{b G} C$ $b(G), f(\operatorname{cl}(\partial(\widetilde{G} \times I)-\widetilde{G} \times\{0\}))=\widetilde{G} \subset G$. Moreover $G \cap \widetilde{\mathrm{hG}}=\partial \widetilde{b G}$ and either $b G \cap$ $\widetilde{G}=\partial \widetilde{G}$ or both $\widetilde{G}$ and $\widetilde{b G}$ are discs.

If $\widetilde{G}$ and $\widetilde{b G}$ are both discs, then denote by $B$ the ball $f(\widetilde{G} \times I)$. One can show that each component of $F$ lies entirely outside this ball. Hence each component of $F$ is contained in the complement of $B$. If $F_{i}$ is a component without boundary, the incompressibility of $F_{i}$ guarantees that it, too, lies outside of $B$.

Thus we can push $\widetilde{b G}$ out across $G$ by an ambient isotopy of $C$ which is fixed outside a small regular neighborhood of $B$. This isotopy reduces the number of intersection curves of $b G$ and $G$.

We now take up a pair $(\widetilde{G}, \widetilde{b G})$ with $G \cap \widetilde{b G}=\partial(\widetilde{b G})$ and $b G \cap \widetilde{G}=\partial \widetilde{G}$ and such that $\widetilde{G} \neq G$. Denote by $D$ the set $f(\widetilde{G} \times I)$. By [13, Corollary 3.2$]$ we see that any closed component $F_{i}$ of $F$ must lie outside of $D$. The same argument, used when $\widetilde{G}$ is a disc, shows that each $F_{i}$ with nonvacuous boundary lies outside of $D$, too. Therefore, there is an isotopic deformation of $b G$ fixed on $\mathrm{cl}(b G-\widetilde{b G})$ and on $F \cup C$ which takes $\widetilde{b G}$ onto $\widetilde{G}$. Now push off $\widetilde{b G}$ slightly to the other side of $G$. Again we have reduced the number of intersection curves of $G \cap b G$. Since the number is finite this procedure terminates with $G \cap b G=$ $\varnothing$. We get $f(\widetilde{G} \times\{0\})=G$ and consequently $\widetilde{b G}=b(G)$. Recalling that $f$ is an embedding, $f(\widetilde{G} \times I)=D$ is a manifold in $C$ bounded by $G \cup b G$. If $F_{i}$ is a component of $F$ with nonvacuous boundary, then $F_{i}$ lies outside of $D$ since $F \cap \partial D=F \cap(G \cup b(G))=\varnothing$. If $F_{i}$ is closed and is contained in $D$, then by [13, Corollary 3.2] $F_{i}$ is parallel to $G$.

This cannot occur, for if $\rho$ is a simple arc in the region bounded by $G$ and $F_{i}$ with an endpoint on each of $G$ and $F_{i}$, then $b \rho$ has its endpoints on each of $b(G)$ and $F_{i}$. But $b$ does not interchange the sides of $F_{i}$.

We have shown that all components of $F$ lie outside of $D$. This completes the proof.

2. Homotoping $H$ to projection onto a surface. In order to isotope $b$ to the identity we will use certain theorems that say roughly, "If the homotopy $H$ has certain properties, then we can isotope $b$ to a "nicer map." " In particular, we 
will see that if the restriction of $H$ to a surface $F$ is projection onto $F$, then we will be able to construct isotopies which are fixed on $F$.

A homotopy $H: X \times I \rightarrow X$ is called projection onto $X$ or projection onto the first factor if $H(x, s)=x$ for every $x$ in $X$ and $s$ in $I$.

In the first part of this section we answer the question: If $b$ is the identity on $\partial M$, when and how can we homotope $H$ so that $H \mid \partial M \times I$ is projection onto $\partial M$ ? (Cf. Propositions 2.1, and 2.2.)

The second and third parts prepare the way for surfaces in M; Part 2 for closed surfaces $F$ and Part 3 for surfaces with nonvacuous boundary.

Part 1. $F$ is a boundary component. If $X$ and $Y$ are spaces and $f: X \rightarrow Y$ is a map we say $f$ is a proper map if for every compact subset $C$ of $Y, f^{-1}(C)$ is compact in $X$. Given a homotopy $H: X \times I \rightarrow Y$, for each $t$ in $I$ we define the map $H_{t}: X \rightarrow Y$ by $H_{t}(x)=H(x, t) . H$ is a proper homotopy means that $H$ is a proper map not just that $H_{t}$ is proper for each $t$.

Proposition 2.1. Let $F$ be a surface with vacuous boundary, different from a plane, an open annulus or a torus. Let $H: F \times I \rightarrow F$ be a bomotopy with $H_{0}=H_{1}=$ identity. Then there is a bomotopy $K: F \times I \times I \rightarrow F$ rel $F \times \partial I$ of $H$ to projection onto $F$. If $H$ is a proper map, then $K$ may be chosen to be proper.

Before proving Proposition 2.1 let us discuss the three excluded cases. We wish to define the homotopy $K: F \times I \times I \rightarrow F$ of $H$ to projection on to $F$. $K$ must be fixed on $F \times \partial I$. Thus $K$ is already given on $(F \times I \times\{0\}) \cup(F \times I \times\{1\}) \cup$ $(F \times \partial I \times I)$. Therefore if $x$ is in $F, K$ is defined on $\partial(\{x\} \times I \times I)$. We can extend $K$ to all of $\{x\} \times I \times I$ only if the loop $K \mid \partial(\{x\} \times I \times I)$ is not null homotopic. If $F$ is an open annulus or a torus, it may happen that $K \mid \partial(\{x\} \times I \times I)$ is not null homotopic. For example if $F$ is an open annulus and $\lambda$ is a simple closed curve representing the generator of $\pi_{1}(F)$, then $H$ could be rotation of the annulus $n$ times around in the direction of $\lambda$. Similarly for the torus. For the plane, $K$ would exist for a similar rotation but could not be chosen proper. For these spaces we have the weaker Proposition 2.2 which first "undoes" the rotation by an isotopy which goes $n$ times around in the opposite direction. Precisely, we get

Proposition 2.2. Let $F$ be a plane, a torus, or an open annulus. Let $H$ : $F \times I \rightarrow F$ be a bomotopy (proper bomotopy) sucb that $H_{0}=H_{1}=$ identity. Then there is an isotopy $J: F \times I \rightarrow F$ of the identity to itself so that " $H$ followed by $J$ " is bomotopic (proper bomotopic) rel $F \times \partial l$ to projection onto $F$.

Lemmas 2.3 and 2.4 prove Proposition 2.1 for closed surfaces.

Lemma 2.3. Let $F$ be a closed surface different from a torus and $H: F \times I$ 
$\rightarrow F$ be a bomotopy of the identity to itself. Then for any $x$ in $F$, the loop $H \mid\{x\} \times I$ is null bomotopic.

Proof. Let $a$ be in $\pi_{1}(F, x)$. Let $f$ represent $\alpha$. Denote by $\gamma$ the homotopy class of $H .\{x\} \times I$. Define $g: I \times I \rightarrow F$ by $g(s, t)=H(f(s), t)$ for $s$ and $t$ in $I$. The homotopy class of the loop $g \mid \partial(I \times I)$ is the identity of $\pi_{1}(F, x)$. But

$$
[g \mid \partial(I \times I)]=\alpha \gamma \alpha^{-1} \gamma^{-1} \text {. }
$$

Hence $1=[g \mid(\partial I \times I)]=\alpha y \alpha^{-1} \gamma^{-1}$. But $\alpha$ was arbitrary. Therefore a lies in the center of $\pi_{1}(F, x)$. Since $F$ is not a torus, the center of $\pi_{1}(F, x)$ is trivial. Thus $H \mid\{x\} \times I$ is null homotopic.

Lemma 2.4. Let $F$ be a closed surface and let $H: F \times I \rightarrow F$ be a bomotopy of the identity to itself. If there is a point $x_{0}$ in $F$ such that the loop $H \mid\left\{x_{0}\right\}$ $\times I$ is null bomotopic then $H$ is bomotopic rel $F \times \partial I$, to projection onto $F$.

Proof. Let $\lambda$ be a simple closed curve containing $x_{0}$. Assume $\lambda$ is not trivial if $F$ is not a 2-sphere. In the case of the 2-sphere the proof is Step 1 followed by Step 4 .

Step 1. We assert that there is a homotopy $K$ of $H$ rel $F \times \partial I$ to a map $K_{1}$ such that $K_{1} \mid \lambda \times I$ is projection onto $\lambda$. As we define $K$ we will show where we may choose the range of $K$. Although this is not necessary when $F$ is compact, we will need this kind of information when we consider the noncompact case.

Let $U(\lambda)$ be a regular neighborhood of $\lambda$. We begin by defining $K$ on

$$
(F \times \partial I \times I) \cup(F \times I \times\{0\}) \cup(\lambda \times I \times\{1\}) \cup(\mathrm{cl}(F-U(\lambda) \times I \times I) .
$$

For any $s$ and $t$ in $l$, let

$$
\begin{array}{ll}
K(x, s, 0)=H(x, s) & \text { for } x \text { in } F, \\
K(x, 0, t)=K(x, 1, t)=x & \text { for } x \text { in } F, \\
K(x, s, 1)=x & \text { for } x \text { in } \lambda, \\
K(x, s, t)=H(x, s) & \text { for } x \text { in } \operatorname{cl}(F-U(\lambda)) .
\end{array}
$$

We show now that we can define $K$ on $\lambda \times I \times I . K$ is already defined on the loop $\partial\left(x_{0} \times I \times I\right)$. Since $H \mid x_{0} \times I$ is null homotopic the loop $K \mid \partial\left(x_{0} \times I \times I\right)$ is null homotopic. Thus we can extend $K$ to all of $\left\{x_{0}\right\} \times I \times I$. The complement of $x_{0} \times I \times I$ in $\lambda \times I \times I$ is an open 3-cell. We can extend $K$ over the rest of $U(\lambda) \times I \times I$ by using the homotopy extension theorem.

Step 2. We start afresh; now assume that $H$ is given so that $H \mid \lambda \times I$ is projection onto $\lambda$. We can homotope $H$ to a map which is projection onto the first factor in a chosen regular neighborhood $U^{\prime}(\lambda)$. (Omit this step for the 2-sphere.) 
Applications of the homotopy extension theorem and simple obstruction theory arguments will produce a homotopy $K$ of $H$ to the desired map. One can insure that $K\left(U^{\prime}(\lambda) \times I \times I\right) \subset H\left(U^{\prime}(\lambda) \times I\right)$.

Step 3. (Omit for the 2-sphere.) Let $P$ be a collection of properly embedded disjoint arcs in $\mathrm{cl}(F-U(\lambda))$ so that $F-[\mathrm{cl}(F-U(\lambda)) \cup\{\rho \mid \rho$ is in $P\}]$ is an open disc. We assume $H \mid U(\lambda) \times I$ is projection onto $U(\lambda)$ and construct a homotopy $K$ rel $F \times \partial I$ of $H$ to a map $K_{1}$ so that $K_{1} \mid U(\lambda) \cup\{\rho \mid \rho \in P\}$ is projection onto the first factor. Again the argument is simple and again we can choose $K$ so that $K(\rho \times I \times I) \subset H(\rho \times I)$.

Step 4. We now assume $H$ is projection onto the first factor on $A=U(\lambda) \cup$ $\{\rho \mid \rho \in P\} . \mathrm{cl}(F-A)$ is a disc with some boundary points identified. We define a homotopy $K^{\prime}: \Delta \times I \times I \rightarrow H(\Delta \times I)$ rel $\partial(\Delta \times I)$ of $H$ to projection onto $\Delta$ subject to the appropriate restrictions.

The homotopy $K: F \times I \times I$ defined by $K_{t} \mid A \times I=H$ and $K_{t} \mid \Delta \times I=K_{t}^{\prime}$ is a homotopy rel $F \times \partial I$ of $H$ to projection onto $F$. This completes the proof of (2.4).

The preceding two lemmas prove (2.1) for closed surfaces.

Lemmas 2.6 and 2.7 prove Proposition 2.1 for the noncompact case. We will need the following version of the homotopy extension theorem, which is implied by the usual proof.

(2.5) Let $X$ and $Y$ be complexes and let $X^{\prime}$ be a subcomplex of $X$. Suppose $K^{\prime}$ is a proper map of $X \times\{0\} \cup X^{\prime} \times I$ into $Y$. Then there is a proper map $K: X \times I \rightarrow Y$ extending $K^{\prime}$. Furthermore $K$ can be chosen so that $K(X \times I)=$ $K^{\prime}\left(X \times\{0\} \cup X^{\prime} \times I\right)$.

Let $X$ be a noncompact complex. A collection $\left\{X_{i}\right\}$ for $i$ an integer is an exbausting sequence for $X$ if

(1) $X_{i}$ is compact for each $i$.

(2) $X_{i} \subset X_{i+1}^{\circ}$ for each $i$.

(3) $\bigcup_{i=1}^{\infty} X_{i}=X$.

If $X$ is a surface or a manifold there exists an exhausting sequence for $X$ such that

(1') $X_{i}$ is a compact connected manifold (surface) for each $i$.

(2') Each component of $X-X_{i}$ is unbounded for each $i$. We get such an exhausting sequence by first taking regular neighborhoods of each $X_{i}^{\prime}$ of some arbitrary exhausting sequence. Now each $X_{i}^{\prime}$ is a manifold (surface) - though not necessarily connected. Let $X_{1}$ be any component of $X_{1}^{\prime}$. Let $X_{i}$ be the component of $X_{i}^{\prime}$ containing $X_{i-1}$. The reader can check that $\left(X_{i}\right)$ is an exhausting sequence satisfying $\left(1^{\prime}\right)$; we add all bounded components of $X-X_{i}$ to $X_{i}$. One can show this new set is still compact. 
If $H: X \times I \rightarrow X$ is any homotopy, by choosing subsequences of $X_{i}$ we may insure

( $\left.3^{\prime}\right) H\left(X_{i} \times I\right) \subset X_{i+1}^{\circ}$.

If $H: X \times I \rightarrow X$ is a proper homotopy, then again by choosing subsequences we get

( $4^{\prime}$ a) $H\left(\partial X_{i} \times I\right) \subset X_{i+1}^{\circ}-X_{i-1}$.

(4'b) $H^{-1}\left(X_{i}\right) \subset X_{i+1} \times I$.

The statements of Lemmas 2.6 and 2.7 refer to exhausting sequences satisfying $\left(1^{\prime}\right),\left(2^{\prime}\right),\left(3^{\prime}\right)$, and $\left(4^{\prime}\right)$ where applicable.

Lemma 2.6. Let $F$ be a noncompact surface without boundary and assume $F$ is neither a plane nor an open annulus. Let $H: F \times I \rightarrow F$ be a bomotopy of the identity to itself and let $\left\{F_{i}\right\}$ be an exhausting sequence as given above. Moreover, suppose $F_{1}$ is not a disc or an annulus. Then for each $i$ and for each boundary component $\lambda$ of $F_{i}$ and each point $x$ on $\lambda$, the loop $H \mid\{x\} \times I$ is null bomotopic in $H(\lambda \times I)$.

Proof. This proof borrows ideas from Epstein's paper [6]. We remark first that we can apply results from $\left[6\right.$, pp. 91-92] to $\pi_{1}(H(\lambda \times I), x)$ by noting that $H(\lambda \times I)$ is a strong deformation retract of a regular neighborhood of $H(\lambda \times I)$. Let $\lambda$ be a boundary component of $F_{i}$ and let $x$ belong to $\lambda$. Let $H \mid\{x\} \times I$ represent $\gamma$ in $\pi_{1}(H(\lambda \times I), x)$. As in Lemma 2.3 define $g_{\lambda}: I \times I \rightarrow H(\lambda \times I)$ by $g_{\lambda}(s, t)=H(\lambda(s), t)$. As in 2.3 again, $g \mid \partial(I \times I)$ represents $[\lambda] \gamma[\lambda]^{-1} \gamma^{-1}$ in $\pi_{1}(H(\lambda \times I), x)$, so $[\lambda]$ and $\gamma$ lie in the same cyclic subgroup of $\pi_{1}(H(\lambda \times I), x)$. We conclude $\gamma=[\lambda]^{n}$ for some nonnegative integer and show now that $n=0$, i.e., $H \mid\{x\} \times I$ is null homotopic.

If $F_{i}$ has more than one boundary component let $\beta$ be an element of $\pi_{1}\left(F_{i}, x\right)$ represented by a simple closed curve $\mu$ which is homotopic to another boundary curve of $F_{i}$. If $F_{i}$ has only one boundary component let $\beta$ be a generator of $\pi_{1}\left(F_{i}, x\right)$ represented by a meridianal simple closed curve $\mu$. Using the function $g_{\mu}$ we get that $\gamma=[\mu]^{m}$ in $\pi_{1}(H(\mu \times I), x)$. Using the natural maps $\pi_{1}(H(\lambda \times I), x)$ $\rightarrow \pi_{1}\left(F_{i+1}, x\right)$ and $\pi_{1}(H(\lambda \times I), x) \rightarrow \pi_{1}\left(F_{i+1}, x\right)$ we have that, in $\pi_{1}\left(F_{i+1}, x\right)$, $\gamma=[\mu]^{m}$ and $\gamma=[\lambda]^{n}$. But $\pi_{1}\left(F_{i}, x\right) \rightarrow \pi_{1}\left(F_{i+1}, x\right)$ is a monomorphism by $\left(2^{\prime}\right)$ and hence $\gamma=[\lambda]^{n}=[\mu]^{m}$ holds in $\pi_{1}\left(F_{i}, x\right)$. But $[\lambda]^{n}=[\mu]^{m}$ only if $n=m=0$.

Lemma 2.7. Let $F$ be a noncompact surface without boundary. Let $H: F \times$ $I \rightarrow F$ be a bomotopy of the identity to itself and let $\left\{F_{i}\right\}$ be an exhausting se. quence satisfying $\left(1^{\prime}\right)-\left(3^{\prime}\right)$ above. If for each $i$ and every component $\lambda$ of $F_{i}$, there is a point $x$ in $\lambda$ so that the loop $H \mid\{x\} \times I$ is null bomotopic, then $H$ is bomotopic rel $F \times \partial I$ to projection onto $F$. 
If $H: F \times I \rightarrow F$ is a proper homotopy.assume $\left\{F_{i}\right\}$ satisfies $\left(1^{\prime}\right)-\left(4^{\prime}\right)$. Suppose there is a function $n(i)$, such that $n(i)<i$ and that $n(i) \rightarrow \infty$ as $i \rightarrow \infty$, so that for each boundary component $\lambda$ of $F_{i}$ there is a point $x$ in $\lambda$ such that the loop $H \mid\{x\} \times I$ is null homotopic in $F-F_{n(i)}$. Then $H$ is proper homotopic to projection onto $F$.

Proof. We follow the proof of Lemma 2.4 making the necessary modifications for the fact $F$ is noncompact. We construct the homotopy $K: F \times I \times I \rightarrow F$ of $H$ to projection onto $F$.

Step 1. Let $H$ be the homotopy (proper homotopy) given in the hypotheses. There is a homotopy (proper homotopy) $K: F \times I \times I \rightarrow F$ rel $F \times \partial I$ of $H$ to a map $K_{1}$ so that $K_{1} \mid \lambda \times I$ is projection onto $\lambda$ for each boundary loop of each $F_{i}$.

Denote the collection of all boundary loops by $Q$. Let $\{U(\lambda) \mid \lambda \in Q\}$ be a collection of pairwise disjoint neighborhoods. We first define $K$ on $[(F \times \partial I \times I)]$ $\cup[(F \times I \times\{0\})] \cup[(\mathrm{cl}(F-\bigcup\{U(\lambda) \mid \lambda \in Q\}) \times I \times I] \cup[(\{\lambda \mid \lambda \in Q\} \times I \times\{1\})]$. For $s$ and $t$ in $l$, define

$$
\begin{array}{ll}
K(x, s, 0)=H(x, s) & \text { for } x \text { in } F, \\
K(x, 0, t)=K(x, 1, t)=x & \text { for } x \text { in } F, \\
K(x, s, 1)=x & \text { for } x \text { in } \bigcup\{\lambda \mid \lambda \in Q\}, \\
K(x, s, t)=H(x, s) & \text { for } x \text { in } \operatorname{cl}(F-\bigcup\{U(\lambda) \mid \lambda \in Q\}) .
\end{array}
$$

Repeat the argument given in Step 1 of (2.4) for each $\lambda$ in $Q$. This defines the desired $K$, if we do not assume $H$ is proper. If $H$ is proper, we want $K$ to be proper. To see that we can guarantee this we note that for each $\lambda$ of $F_{i}$, there is an $x$ in $\lambda$ such that $H \mid\{x\} \times I$ is null homotopic in $F-F_{n(i)}$. Recalling that the loop $K \mid \partial(\{x\} \times I \times I)$ is homotopic to $H \mid\{x\} \times I$ we can extend $K$ to $\{x\} \times I$ $\times I$ such that $K(\{x\} \times I \times I) \subset F-F_{n(i)}$. To extend $K$ over $\lambda \times I \times I$, the reader can check that the obstruction lies in $\pi_{2}\left(\left(F-F_{n(i)}\right) \cup H(\lambda \times I)\right)$, which is trivial. Hence we can extend $K$ over $\lambda \times I \times I$ so that $K(\lambda \times I \times I) \subset\left(F-F_{n(i)}\right) \cup H(\lambda \times I)$. Now using (2.5), the proper homotopy extension theorem, $K$ can be extended over $U(\lambda) \times I \times I$ so that $K(U(\lambda) \times I \times I) \subset\left(F-F_{n(i)}\right) \cup(H(U(\lambda) \times I))$. It is easily seen that $K$ is a proper map.

We carry out Steps 2, 3 and 4 as in the proof of Lemma 2.4 making the appropriate changes for noncompactness.

Part 2. Closed surfaces.

Proposition 2.8. Let $C$ be a compact irreducible manifold with nonvacuous boundary. Let $F$ be a closed incompressible surface in $C$. Assume that if $F$ separates $C$, then $F$ separates $\partial C$. Let $b: F \rightarrow C$ be a bomeomorpbism of $F$ onto itself and let $H: F \times I \rightarrow C$ be a bomotopy of $b$ to the inclusion function. Then there is a bomotopy of $H$ rel $F \times \partial I$ to a map of $F \times I$ into $F$. 
Proof. Let $U(F \times \partial I)$ be $F \times([0, \epsilon] \cup[1-\epsilon, 1])$ for some $\epsilon<1 / 2$. Identify points in a regular neighborhood $U(F)$ in $C$ with $F \times[-\epsilon, \epsilon]$ so that $x$ in $F$ corresponds to $(x, 0)$. There is a homotopy $H^{\prime}$ which is homotopic rel $F \times \partial I$ to $H$ and has the property that $H^{\prime-1}(F) \cap U^{\prime}(F \times \partial I)=F \times \partial I$ where $U^{\prime}(F \times \partial I)=$ $F \times([0, \epsilon] \cup[1-\epsilon, 1])$. Since it suffices to show that $H^{\prime}$ is homotopic rel $F \times$ $\partial I$ to a map of $F \times I$ into $F$, we will denote $H^{\prime}$ by $H$ and start anew.

Applying the proposition on p. 60 of [13], we can homotope

$$
H \mid \mathrm{cl}\left(F \times I-U^{\prime}(F \times \partial I)\right) \operatorname{rel} \partial\left[\mathrm{cl}\left(F \times I-U^{\prime}(F \times \partial I)\right)\right]
$$

to a map (which we will continue to denote by $H$ ) which is transverse with respect to $F$ and so that $H^{-1}(F) \cap \mathrm{cl}\left(F \times I-U^{\prime}(F \times I)\right)=F_{1} \cup \cdots \cup F_{m}$ where $F_{j}$ is a system of incompressible surfaces in $F \times I$. We may regard this homotopy as a homotopy of $H$ rel $U^{\prime}(F-\partial I)$ so that afterwards $H^{-1}(F) \cap$ $F \times I=F_{1} \cup \cdots \cup F_{m} \cup F \times \partial I$. Each $F_{i}$ is closed and is parallel to $F \times 0$. By a commutative diagram $\left(H \mid F_{i}\right)_{*}$ is an isomorphism. Therefore by [13, Lemma 1.4.3], $H \mid F_{i}$ is homotopic to a homeomorphism. Let $U\left(\bigcup_{i=1}^{m} F_{i}\right)$ denote a regular neighborhood of $\bigcup_{i=1}^{m} F_{i}$. Using the homotopy given above on each side of the regular neighborhood around $F_{i}$ and connecting up to a homotopy which is essentially our original one on $\mathrm{cl}\left(F-U\left(\bigcup_{i=1}^{m} F_{i}\right)\right)$ we can homotope $H$ rel $F \times \partial I$ to a map which we still call $H$ so that $H \mid F_{i}$ is a homeomorphism for each $i$. Moreover, it is still the case that $H^{-1}(F)=F \times \partial I \cup F_{1} U$ $\cdots \cup F_{m}$.

Any two components of $F \times \partial I \cup F_{1} \cup \cdots \cup F_{m}$ bound a domain homeomorphic to $F \times I$. We will denote such a domain by $F \times I^{\prime}$ where $I^{\prime}$ is some subinterval of $I$. Assume we have numbered the $F_{i}$ so that no $F_{j}$ lies in the region bounded by $F_{i} \cup F_{i+1}$. Let $\widetilde{C}$ be the manifold (not necessarily connected) obtained from $C$ by splitting along $F$. For each region bounded by $F_{i} \cup F_{i+1}$, there is a lift-

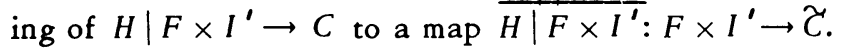

Case 1. Assume $C$ is boundary irreducible.

We have $\operatorname{ker}\left(H \mid F \times I^{\prime}\right)=0$. Furthermore because $F$ separates $\partial C$ whenever $F$ separates $C$, the component of $\widetilde{C}$ containing $\left.\overline{\left(H \mid F \times I^{\prime}\right.}\right)\left(F \times I^{\prime}\right)$ has more boundary components than just $p^{-1}(F)$. Hence using [13, p. 67] $\overline{H \mid F \times I^{\prime}}$ cannot be homotopic to a covering. Therefore each $H \mid F \times I^{\prime}$ is homotopic rel $F \times \partial I^{\prime}$ to a map into $\partial(\widetilde{C})$. It follows that each $H \mid F \times I^{\prime}$ is homotopic rel $F \times \partial I^{\prime}$ to a map into $F$ and hence $H$ is homotopic rel $F \times \partial I$ to a map into $F$.

Case 2. $C$ and hence $\widetilde{C}$ does not have irreducible boundary.

There is a system $\left\{\Delta_{i}\right\}$ of properly imbedded discs in $\widetilde{C}$ so that $\mathrm{cl}\left(\widetilde{C}-U\left(\cup_{i} \Delta_{i}\right)\right)$ is boundary irreducible and so that $H: F \times I \rightarrow \widetilde{C}$ is homotopic rel $F \times \partial I$ to a map into $p\left(\operatorname{cl}\left(\widetilde{C}-U\left(\bigcup_{i} \Delta_{i}\right)\right)\right.$. We are now reduced to the previous case.

Note to Proposition 2.8. Acutally it was unnecessary to assume $F$ is in $C$. 
We could have assumed that $F$ was a component of $\partial C$ such that $F \neq \partial C$. We will use this later.

Lemma 2.9. Let $F$ be a closed surface different from a 2-sphere. Let $H$ : $F \times I \rightarrow F$ be a bomotopy of a bomeomorphism $b$ to the identity. Then $H$ is bomotopic rel $F \times \partial I$ to an isotopy.

Proof. Define $g: F \times I \rightarrow F \times I$ by $g(x, t)=(H(x, t), t)$. Since $g \mid F \times 0$ and $g \mid F \times\{1\}$ are homeomorphisms, by [13, p. 67] there is a homotopy rel $F \times \partial I$ of $g$ to a homeomorphism. Applying [13, p. 69] this last homeomorphism is homotopic rel $F \times \partial I$ to a level-preserving homeomorphism. Compose these homotopies with projection onto the first factor to conclude the proof.

Some of the main results use invariants of proper homotopy type. We make use of the space of ends in the next lemma so we introduce it here. For a more complete exposition see [4].

Let $X$ be a locally-finite complex. Proper maps $b, b^{\prime}:[0, \infty) \rightarrow X$ are said to determine the same end of $X$ provided for $C$ compact and $t$ sufficiently large, $b(t)$ and $b^{\prime}(t)$ are in the same component of $X-C$. This is an equivalence relation and we call the equivalence class of $b$ an end of $X$. The set of ends is denoted by $\underline{\pi}_{0}(X)$.

If $f: X \rightarrow Y$ is a proper map, then $f$ induces (by composition) a map $\underline{\pi}_{0}(f)$ : $\underline{\pi}_{0}(X) \rightarrow \underline{\pi}_{0}(Y)$. If $f$ and $g$ are proper homotopic then $\underline{\pi}_{0}(f)=\underline{\pi}_{0}(g)$.

Lemma 2.10. Suppose $M$ is a noncompact irreducible manifold and $G$ is any closed surface in $M$ different from the 2-sphere. Let $H: M \times I \rightarrow M$ be a proper bomotopy of a bomeomorphism $b$ to the identity such that $H(G \times I)$ is contained in $G$. (In particular $b(G)=G$.) Then $b$ does not interchange the sides of $G$.

Proof. If $M-G$ is connected, let $\lambda$ be a closed curve which intersects $G$ transversally in one point. If $b$ interchanges the sides of $G$, then $b$ will change the intersection number of $G$ with $\lambda$ which contradicts the fact that $b$ is homotopic to the identity.

Suppose $G$ separates $M$. Then $M-G=A \cup B$. Assume $B$ is unbounded. If $A$ is bounded, clearly $b(A)=A$. If $A$ is unbounded, we argue as follows. Since $b$ is proper homotopic to the identity, $\underline{\pi}_{0}(b)$ is the identity and hence maps ends of $A$ to themselves. Again $b(A)=A$. Thus in either case $b$ does not interchange the sides of $G$.

Part 3. Compact surfaces. The next three lemmas are imbedded in proofs from [13].

Lemma 2.11. Let $M$ be an irreducible manifold and $H: M \times I \rightarrow M$ be a bomotopy of a bomeomorphism $b$ to the identity. Suppose $H \mid \partial M \times I$ is projection onto $\partial M$. 
Let $F$ be a system of compact surfaces in $M$ which are properly imbedded and incompressible in some compact irreducible submanifold $C$ of M. For each component $F_{i}$ of $F$ assume $h\left(F_{i}\right)=F_{i}$.

Suppose $G$ is a compact surface with nonvacuous boundary in $M$ such that $G$ is properly imbedded and incompressible in $C$. Assume also that $G \cap F=\varnothing$. Suppose $H(G \times I)$ is contained in $C$.

Then there is a bomotopy of $H \mid G \times I \mathrm{rel} \partial(G \times I)$ to a map of $G \times I$ into $C-U(F)$, where $U(F)$ is some chosen regular neighborbood of $F$.

Lemma 2.12. Let $G$ be a compact incompressible surface with nonvacuous boundary in an irreducible manifold $C$. Let $b$ be a bomeomorphism of $G$ onto itself and let $H: G \times I \rightarrow C$ be a map with $H_{0}=b, H_{1}=$ inclusion and $H \mid \partial G \times I=$ projection onto $G$. Then there is a bomotopy of $H$ rel $\partial(G \times I)$ to a map which takes $G \times I$ into $G$.

Lemma 2.13. Let $G$ be a compact surface with nonvacuous boundary. Let $H: G \times I \rightarrow G$ be a bomotopy rel $\partial G$ of the identity to itself. Then $H$ is homotopic rel $\partial(G \times I)$ to projection onto $G$.

Lemma 2.14. Let $M$ be an irreducible manifold and $F$ a compact surface in $M$ with or without boundary. Suppose $U(F)$ and $U^{\prime}(F)$ are regular neighborboods of $F$ in $M$ with $U^{\prime}(F)$ contained in the interior of $U(F)$. Let $H: M \times I \rightarrow M$ be a bomotopy of a bomeomorphism $b$ to the identity. Suppose $b \mid U^{\prime}(F)$ is the identity, $H \mid F \times l$ is projection onto $F$, and $H \mid \partial M \times I$ is projection onto $\partial M$. Then there is a bomotopy $K: M \times I \times I \rightarrow M$ of $H$ rel $\partial(M \times I) \cup(F \cup \operatorname{cl}(M-U(F))) \times I$ to a bomotopy. $H^{\prime}$ such that $H^{\prime} \mid U^{\prime}(F) \times I$ is projection onto $U^{\prime}(F)$.

Proof. By setting up the desired end result and using the homotopy extension theorem one can produce the homotopy one needs.

3. The Main Lemma and the isotopy result for eventually end-irreducible manifolds. The isotopy results we have proved are for noncompact irreducible manifolds which are either end-irreducible or eventually end-irreducible. As mentioned in the introduction, end-irreducible is a condition on ends playing a role similar to the condition of incompressible for boundary components. By removing some boundary components of compact manifolds with irreducible boundary, one gets many examples of end-irreducible manifolds. Nontrivial examples will be constructed later in this section.

Since the definition of end-irreducible involves defining the new proper fundamental groups of E. M. Brown and since we use only the facts given in (3.1) below about end-irreducible manifolds the reader is referred to [4] and [5] for definitions.

Let $X$ be a subcomplex of $Y$. So as to avoid confusion we (in the case $Y$ 
is a manifold) use the word frontier of $X$ to mean $\mathrm{cl}(X)-X$, and we write $\mathrm{Fr}_{\mathrm{r}}(X)$. If $C$ is a compact submanifold of a manifold $M$ we say $C$ is frontier-incompres. sible if each component to $\operatorname{Fr}(C)$ is an incompressible surface in $M$.

(3.1) [5, to appear]. Let $M$ be an end-irreducible manifold. There exists an exhausting sequence $\left\{C_{n}\right\}$ for $M$ sucb that

(1) $C_{n}$ is a compact submanifold of $M$.

(2) $\mathrm{Fr}_{\mathrm{r}}\left(C_{n}\right)$ is a system of properly imbedded surfaces in M meeting no compact component of $\partial M$.

(3) $\operatorname{Fr}\left(C_{n}\right)$ is incompressible in $M$.

(4) $C_{n}$ is connected.

(5) All components of $M-C_{n}$ are unbounded.

Finally for the notion of a bierarchy for an irreducible compact manifold $D$, we follow Waldhausen in [13]. Notice that (3) of (3.1) and the fact that any compact irreducible manifold with nonvacuous boundary has a hierarchy gives us an infinite hierarchy for an irreducible and end-irreducible manifold.

In the proofs presented in the next two sections, a key theorem is

(3.2) [13, p. 76]. Let $C$ be a compact irreducible manifold. Let $F$ and $G$ be incompressible surfaces in $C$. Suppose there is a bomotopy carrying $F$ onto $G$ which is constant on $\partial F$. Then there is an ambient isotopy of $C$ carrying $F$ onto $G$ which is constant on $\partial C$.

Main Lemma. This proposition forms the heart of the proofs of all the isotopy results which follow. It allows us to reduce the proofs to Alexander's theorem by showing that one can isotope $b$ to the identity on part of a hierarchy without changing the homeomorphism where it has already been isotoped to the identity.

Proposition 3.3. Let $M$ be a noncompact irreducible manifold and $C$ be $a$ compact submanifold of $M$. Let $D$ be a compact irreducible manifold in $M-C$ sucb that $\operatorname{Fr}(U(D))$ is a system of incompressible surfaces in $M-C$.

Suppose $H: M \times I \rightarrow M$ is a bomotopy of a bomeomorphism $b$ to the identity. Assume $H$ restricted to $\partial M \times I \cup U\left(\mathrm{~F}_{\mathrm{r}}(D)\right) \times I$ is projection onto the first factor, for some regular neighborbood $U\left(\mathrm{~F}_{\mathrm{r}}(D)\right)$ of $\mathrm{Fr}_{\mathrm{r}}(D)$ in $\cdot M-C$. Moreover assume $H(D \times I) \subset M-C$.

Suppose $D_{j}, E_{j} \subset D, U\left(E_{j}\right) \subset D_{j}, D_{j+1}=\mathrm{cl}\left(D_{j}-U\left(E_{j}\right)\right.$ for $j=1, \cdots, n$ is a bierarchy for $D_{1}=\mathrm{cl}\left(D-U\left(\mathrm{~F}_{\mathrm{r}}(D)\right)\right)$.

Then

(1) there is an isotopy $J: M \times I \rightarrow M$, fixed on $\partial M$ and on $(M-D) \cup$ $U(\operatorname{Fr}(D))$, with $J_{1}=b$ and so that $J_{0} \mid U\left(E_{j}\right)$ is the identity for each $j$.

(2) "J followed by $H$ " is bomotopic rel $\partial(M \times I) \cup(M-D) \times I \cup U(\operatorname{Fr}(D))$ $\times I$ to a map whose restriction to $U\left(E_{j}\right) \times I$ is projection onto $U\left(E_{j}\right)$ for each $j$. 
Proof. We begin by showing there is an ambient isotopy of $M$ taking $E_{1}$ onto $b\left(E_{1}\right)$ which is fixed on $\partial M \cup(M-D) \cup U\left(\mathrm{Fr}_{\mathrm{r}}(D)\right)$.

First we show that $H \mid E_{1} \times I$ is homotopic rel $\partial\left(E_{1} \times I\right)$ to a map in to $\operatorname{cl}\left(D-U\left(F_{\mathrm{r}}(D)\right)\right)$.

Denote by $F$ the system $\mathrm{Fr}_{\mathrm{r}}(D)$ of incompressible surfaces in $M-C$. $H \mid \partial\left(E_{1} \times I\right)$ is trivially transverse with respect to $F$. Thus by $[13$, p. 60] there is a homotopy of $H \mid E_{1} \times I$ rel $\partial\left(E_{1} \times I\right)$ after which $H_{E_{1}^{-1}}^{-1}(F)$ is a system of incompressible surfaces in $E_{1} \times I$. ( $H_{E_{1}}$ denotes $H \mid E_{1} \times I$.) On the one hand, these surfaces must be closed because $H\left(\partial\left(E_{1} \times I\right)\right) \cap F=\varnothing$. On the other hand, since $E_{1}$ has boundary each surface in $H_{E_{1}^{-1}}^{-1}(F)$ must have boundary. Hence $H_{E_{1}}^{-1}(F)=\varnothing$. It follows that $H\left(E_{1} \times I\right)$ is contained in $D-F$. Now push $H\left(E_{1} \times I\right)$ out of $U(F)$ and into $\operatorname{cl}\left(D-U\left(\mathrm{Fr}_{\mathrm{r}}(D)\right)\right)$.

Since $b \mid U(\operatorname{Fr}(D))$ is the identity and $b$ globally is a homeomorphism, we have $b\left(E_{1}\right)$ is contained in and is incompressible in $\operatorname{cl}(D-U(\operatorname{Fr}(D)))$. Applying (3.2) now to $H_{E_{1}}: E_{1} \times I \rightarrow \operatorname{cl}\left(D-U\left(\mathrm{Fr}_{\mathrm{r}}(D)\right)\right), E_{1}$ and $b\left(E_{1}\right)$, we get an ambient isotopy of $\operatorname{cl}\left(D-U\left(\mathrm{~F}_{\mathrm{r}}(D)\right)\right)$, fixed on $\partial\left(\mathrm{cl}\left(D-U\left(\mathrm{~F}_{\mathrm{r}}(D)\right)\right)\right.$, which takes $E_{1}$ onto $b\left(E_{1}\right)$. Extending this isotopy to all of $M$ by making it fixed elsewhere gives us the ambient isotopy of $M$ taking $E_{1}$ onto $b\left(E_{1}\right)$. We change $b$ and $H$ according to this isotopy and we begin again assuming that in addition to satisfying the hypotheses of the theorem, $b\left(E_{1}\right)$ and $H\left(E_{1} \times I\right)$ are contained in $\mathrm{cl}(D-U(\mathrm{Fr}(D)))$.

Applying (2.12) with $E_{1}=G, H \mid E_{1} \times I=H$, and $b \mid E_{1}=b$ we can homotope $H \mid E_{1} \times I$ rel $\partial\left(E_{1} \times I\right)$ so that afterwards $H_{E_{1}}\left(E_{1} \times I\right)$ is contained in $E_{1}$. Thus $b \mid E_{1}$ is homotopic in $E_{1}$ to the identity. We can now use [6, p. 99] to get an isotopy on $\operatorname{cl}\left(D-U\left(\mathrm{Fr}_{\mathrm{r}}(D)\right)\right)$ to a map which is the identity on a chosen regular neighborhood $U\left(E_{1}\right)$; moreover this isotopy can be chosen fixed outside a neighborhood $U^{\prime}\left(E_{1}\right)$ containing $U\left(E_{1}\right)$ in its interior and it is fixed on $\partial(\operatorname{cl}(D-U(\operatorname{Fr}(D))))$. We can trivially extend this isotopy to all of $M$. Again we change $b$ and $H$ according to this isotopy and we continue, but assume now in addition that $b \mid U\left(E_{1}\right)$ is the identity where $U\left(E_{1}\right)$ is a regular neighborhood of $E_{1}$ in $\operatorname{cl}(D-U(\operatorname{Fr}(D)))$.

By (2.13), $H \mid E_{1} \times I$ is homotopic rel $\partial\left(E_{1} \times I\right)$ to projection onto $E_{1}$. Construct a homotopy $K$ of $H: M \times I \rightarrow M$ rel $M \times \partial I \cup \mathrm{cl}\left(M-U\left(E_{1}\right)\right) \times I$ to a map $K_{1}$ (which we will still call $H$ ), so that $H \mid E_{1} \times I$ is projection onto $E_{1}$. By (2.14) we get that $H \mid U\left(E_{1}\right) \times I$ is projection onto $U\left(E_{1}\right)$.

Summarizing, we have isotoped $b$ so that $h \mid U\left(E_{1}\right)$ is the identity and homotoped $H$ so that $H \mid U\left(E_{1}\right) \times I$ is projection onto $U\left(E_{1}\right)$. Moreover we have chosen the isotopies and homotopies carefully enough so that the hypotheses of (3.3) still hold. Replacing $D$ by $D_{2}=\operatorname{cl}\left(D-U\left(E_{1}\right)\right)$, we note that all the hypotheses of (3.3) are still satisfied. Hence by induction on the length of the hierarchy for $D$ we are done. 
We come now to the main results of this section. The proof uses Waldhausen's isotopy theorem for compact manifolds with nonvacuous boundary. See [13, p. 81].

If $M$ is Euclidean 3-space the correct version of the isotopy result appears in Epstein's paper:

(3.5) [6, p. 96]. Let $b: R^{3} \rightarrow R^{3}$ be an orientation preserving homeomorphism of Euclidean 3-space. Then there is an isotopy of $b$ to the identity. (This is the same as saying $b$ is proper bomotopic to the identity.)

Our results in this section pertain to eventually end-irreducible manifolds. A noncompact manifold $M$ is eventually end-irreducible if $M \neq R^{3}$ and there exists a compact submanifold $D$ such that $M-D$ is end-irreducible. Removing the boundary from a compact 3-manifold gives one an eventually end-irreducible manifold. A more significant class of manifolds which are all eventually end-irreducible are the uncountably many different contractible open subsets of $R^{3}$ constructed by McMillan in [11]. These manifolds are pattemed after the one described by Whitehead [14] as a counterexample to his "proof" of the Poincaré conjecture. It can be constructed as follows: Let $f: R^{3} \rightarrow R^{3}$ be a homeomorphism of $R^{3}$ such that $f\left(T_{0}\right)=T_{1}$ where $T_{0}$ and $T_{1}$ are solid tori and $T_{0}$ is linked inside of $T_{1}$. Then $M=\bigcup_{i} f_{i}\left(T_{0}\right)$ is a "Whitehead" example. The above function $f: M \rightarrow M$ shows that the assumption in 3.6 is necessary. Since $M$ is contractible $f$ is homotopic to the identity. By the construction given in [17] $f$ cannot be proper homotopic to the identity (and hence it is not isotopic to the identity).

Theorem 3.6. Let $M$ be an irreducible, eventually end-irreducible manifold. Let $b: M \rightarrow M$ be a bomeomorphism which is proper bomotopic to the identity via the map $H: M \times I \rightarrow M$. Suppose that $H(\partial M \times I) \subset \partial M$. Then $b$ is isotopic to the identity.

If $H \mid \partial M \times I$ is projection onto $\partial M$, then the isotopy from $b$ to the identity may be chosen constant on $\partial M$.

Proof. Step 1. Changing $b$ and $H$ on the boundary. Since $H \mid \partial M \times I$ is a proper map, by $[6$, p. 99] there is an isotopy $J: \partial M \times I \rightarrow \partial M$ of $b \mid \partial M$ to the identity. By results in $\$ 2$ we may continue the proof assuming that $H: M \times I \rightarrow M$ is proper homotopic rel $M \times \partial I$ to a map whose restriction to $\partial M \times I$ is projection onto $\partial M$.

Step 2. Changing $H$ and $b$ on a single component $F$ of $\operatorname{Fr}_{\mathrm{r}}\left(C_{2 n}\right)$. Choose a compact submanifold $C_{0}^{\prime}$ of $M$ such that $M-C_{0}^{\prime}$ is end-irreducible. Let $C_{0}=$ $C_{0}^{\prime} \cup b\left(C_{0}^{\prime}\right)$. Now choose an exhausting sequence $\left\{C_{1}^{\prime}\right\}, i=1,2, \cdots$, satisfying (3.1) for each component of $M-C_{0}$. In addition assume the $C_{i}^{\prime \prime}$ 's have been chosen so that 
(a) $H\left(\mathrm{Fr}_{\mathrm{r}}\left(C_{i}^{\prime}\right) \times I\right) \subset C_{i+1}^{0 \prime}-C_{i}^{\prime}$,

(b) $H\left(\left(M-C_{1}^{\prime}\right) \times I\right) \cap C_{0}=\varnothing$,

(c) $H\left(C_{i}^{\prime} \times I\right) \subset C_{i+1}^{\circ \prime}$.

Let $C_{i}=C_{i}^{\prime} \cup C_{0}$ for $i=1,2, \ldots$ (a), (b) and (c) above hold when we replace the $C_{i}^{\prime \prime}$ 's by the $C_{i}^{\prime}$ s.

(1) We denote $\mathrm{cl}\left(C_{2 n+1}-C_{2 n-1}\right)$ by $S_{n}$. Let $F$ be a component of Fr $C_{2 n}$. We show there is an isotopy on $M$ of $b$ rel $\mathrm{cl}\left(M-S_{n}\right) \cup \partial M$ to a homeomorphism (which we continue to denote by $b$ ) so that $b(F)=F$.

The $C_{i}^{\prime \prime}$ 's were chosen so that $H(F \times I) \subset S_{n}$. Hence by (3.2) there is an isotopy of $S_{n}$, fixed on $\partial S_{n}$ carrying $b(F)$ onto $F$. Extending this isotopy so that it is constant outside $S_{n}$, we change $b$ via this isotopy so that now $b(F)=F$.

(2) Let $F$ be a closed component of $\operatorname{Fr}\left(C_{2 n}\right)$. Next we show there is an isotopy of $b$ to a homeomorphism which is the identity on $F$.

We will prove the above by changing $H$ until we are in a position to isotope b. Let $H_{F}$ denote the map $H \mid F \times I$. First, there is a homotopy of $H_{F}$ constant on $F \times \partial I$ after which $H_{F}(F \times I)$ is contained in $F$. This follows from (2.8) with $S_{n}=C$. After this change of $H_{F}$, we apply (2.9) to homotope $H_{F}$ rel $F \times \partial I$ to an isotopy $J: F \times I \rightarrow F$ of $b \mid F$ to the identity. One can now construct a proper homotopy $K: M \times I \times I \rightarrow M$ rel cl $\left(M-S_{n}\right) \times I \cup \partial(M \times I)$ of $H$ to a homotopy $K_{1}$ : $M \times I \rightarrow M$ so that $K_{1} \mid F \times I=J$. Hence we have $K_{1}: M \times I \rightarrow M$, a homotopy of $b$ to the identity, which agrees with $H$ on $\partial M$ and outside of $S_{n}$. Moreover $K_{1} \mid F \times I$ is an isotopy of $b \mid F$ to the identity of $F$. It follows, now, from (2.10) that $b$ does not interchange the sides of $F$. Thus given a regular neighborhood $U(F), b$ is isotopic rel $\mathrm{cl}(M-U(F))$ to a homeomorphism $b^{\prime}$ so that $b^{\prime} \mid F$ is the identity. Moreover $b^{\prime}$ is homotopic to the identity via a homotopy $H^{\prime}: M \mid I \rightarrow M$ so that $H^{\prime} \mid F \times I$ is projection onto $F$. We start afresh with $b^{\prime}$ and $H^{\prime}$ as our new $b$ and $H$.

Step 3. Changing $b$ and $H$ on $U(F)$. In order to prepare the way for future isotopies, change $b$ to be the identity in a chosen neighborhood $U(F)$ and $H$ to be projection there.

At this point we will isotope $b$ to be the identity on the other components of $\operatorname{FrC} 2 n$.

Step 4. Istoping $b$ to the identity on all closed components of $\mathrm{Fr}_{\mathrm{r}} C_{2 n}$. Let $G$ be another closed component of $\mathrm{Fr}_{2 n}$. Applying (1.1) with $C=S_{n}$, we can perform an isotopy of $b$ rel cl $\left(M-S_{n}\right) \cup U^{\prime}(F)$ so that afterwards $b(G)=G$. We may now apply (2.8) to $H_{G}: G \times I \rightarrow S_{n}$ to homotope $H_{G}$ rel $G \times \partial I$ to a map whose image is contained in $G$. We can now proceed from Step 2(2) using regular neighborhoods $U(G)$ and $U^{\prime}(G)$ of $G$ in $S_{n}$ such that $U^{\prime}(G) \subset U(G)$ and $U(G) \cap U(F)$ $=\varnothing$.

We have a new homeomorphism $b$ which is the identity on $U^{\prime}(G)$ and a new 
homotopy $H$ which on $U^{\prime}(G) \times I$ is projection onto $U^{\prime}(G)$. Since $U^{\prime}(G) \cap$ $\left(\partial M \cup U^{\prime}(F)\right)=\varnothing$ we note that $b$ and $H$ have not been changed on $\partial M \cup U^{\prime}(F)$. Moreover since $U(G)$ is contained in $S_{n}$ we have not changed $b$ and $H$ outside of $S_{n}$. Thus by induction we can conclude that $b$ is the identity on a regular neighborhood of the union of the closed components of $\operatorname{Fr}_{2 n}$.

Step 5. Isotoping $b$ to the identity on components with boundary. We need to carry out a similar process for components of $\mathrm{Fr} C_{2 n}$ which are not closed. These components meet the noncompact boundary components of $\partial M$. Let $G$ be one such component. Denote by $F$ the system of closed components of Fr $C_{2 n}$ and let $U(F)$ denote a regular neighborhood of $F$ on which $H$ is projection.

(1) We assert there is an isotopy on $M$ fixed on $U(F) \cup \mathrm{cl}\left(M-S_{n}\right) \cup \partial M$ which carries $b(G)$ onto $G$. By (2.11), with $S_{n}=C$ and $F=$ all closed components of $\mathrm{Fr}_{2 n}$ there is a homotopy of $H_{G}$ rel $\partial(G \times I)$ so that afterwards $H_{G}(G \times I)$ is contained in $S_{n}-U(F)$. Using (3.2) we have an isotopy on $\operatorname{cl}\left(S_{n}-U(F)\right)$, fixed on $\left(\mathrm{cl}_{n}\left(S_{n}-U(F)\right)\right)$, which carries $b(G)$ onto $G$. Extend this isotopy trivially to all of $M$, and our assertion follows.

(2) Changing $b$ and $H$ by this isotopy we proceed assuming $b(G)=G$. Using a procedure similar to the one used for closed components, there is an isotopy of $b$ fixed on $(M-U(G)) \cup \partial M$ to a homeomorphism which is the identity on $U^{\prime}(G)$. The results of Part $3, \S 2$ give us a homotopy of $H$ to a map which is the projection map when restricted to $U^{\prime}(G)$. It can be done so that $H$ is not changed on $(M-U(G)) \times I \cup \partial(M \times I)$.

It is clear that (2.11) permits us to make an induction on the number of compact surfaces with boundary in $\operatorname{Fr} C_{2 n}$. We conclude that, for each positive integer $n, H$ is proper homotopic rel cl $\left(M-S_{n}\right) \times I \cup \partial M \times I$ to a map $H_{n}^{\prime}: M \times I$ $\rightarrow M$ such that $H_{n}^{\prime} \mid U\left(\operatorname{Fr}_{2 n}\right) \times I$ is projection onto the first factor. Moreover $\left(H_{n}^{\prime}\right)_{0}$ is isotopic rel cl $\left(M-S_{n}\right) \cup M$ to $b$, and $\left(H_{n}^{\prime}\right)_{1}=$ identity.

Step 6. Fitting together the homotopies and isotopies to bring the proof to an end. There is a well-defined map $H^{\prime}$, where $H^{\prime}=\bigcup_{n} H_{n}^{\prime} \cup H \mid C_{0} \times I$ on $M \times I$. Using the proper homotopy extension theorem for each homotopy of $H$ we have that $H_{n}^{\prime}\left(S_{n} \times I\right) \subset H\left(S_{n} \times I\right)$. This and the fact that $H$ is proper show that $H^{\prime}$ is proper.

Using a similar argument we can show that $b$ is isotopic rel $\partial M$ to a map which is the identity on $U\left(\mathrm{Fr}_{\mathrm{r}} \mathrm{C}_{2 n}\right)$ for every $n$.

We continue with the proof assuming $H \mid U\left(\mathrm{Fr}_{2} C_{2 n}\right) \times I$ is projection onto $U\left(F_{r} C_{2 n}\right)$ for each $n$. By choosing a hierarchy for each shell one can apply Proposition 3.3 and obtain a homotopy of $b$ to a map which is the identity on all but a collection of disjoint open cells of $M-C_{2}$. Let $\Delta$ be the closure of one of the above open cells. $b \mid \partial \Delta$ is the identity. Therefore by Alexander's theorem, 
$b \mid \Delta$ is isotopic rel $\partial \Delta$ to the identity. Thus we may isotope $b$ rel $\partial \Delta$, for all such cells $\Delta$, to a homeomorphism which is the identity on $\mathrm{cl}\left(\mathrm{M}-\mathrm{C}_{2}\right)$. Also there is no obstruction to homotoping $H$ so that it will be the projection map when restricted to one of these cells.

At this point $b \mid \mathrm{cl}\left(M-C_{2}\right)=$ identity and $H \mid \mathrm{cl}\left(M-C_{2}\right) \times I=$ projection onto the first factor. To finish the proof, we note that since we chose the $C_{i}$ 's so that $H\left(C_{i} \times I\right)$ is contained in $C_{i+1}, H\left(C_{3} \times I\right)$ is contained in $C_{3} ; H \mid \partial\left(C_{3}\right)$ $\times I$ is projection onto $\partial C_{3}$. Therefore, by [13, Theorem 7.1], $b \mid C_{3}$ is isotopic rel $\partial C_{3}$ to the identity. Extending this isotopy trivially to all of $M$ we have that $b$ is isotopic to the identity.

Corollary 3.7. Let $M$ be a noncompact irreducible, eventually end-irreducible manifold with compact, irreducible boundary components. Let $H: M \times I \rightarrow M$ be a proper homotopy of a bomeomorphism $b$ to the identity. Then $b$ is isotopic to the identity.

Proof. See the note following (2.8) to reduce ourselves to (3.6).

4. Isotopy results for end-irreducible manifolds and a twisted $I$-bundle theorem. For the next theorems we will not assume that $H$ is proper. As shown in the example given in $\$ 3$, we must make some additional hypotheses on the manifold. We assume that $M$ is end-irreducible.

Lemma 4.2 is our replacement of (2.10). For this we need Brown and Tucker's generalization of [13, Theorem 6.1].

(4.1) [5, to appear]. Let $M$ and $N$ be irreducible, boundary-irreducible, and end-irreducible manifolds. Suppose $N$ is noncompact but that boundary components of $M$ are compact. Finally suppose there is a proper map $f:(M, \partial M) \rightarrow(N, \partial N)$ so that $\pi_{1}(f)$ is a monomorphism. Then there is a proper bomotopy of $f$ (as a map of pairs) to a map $f_{1}$ so that either (a) or (b) bolds:

(a) $f_{1}$ is a finite sheeted covering map.

(b) There are closed surfaces $F$ in $M$ and $G$ in $N$ so that $M$ is a vector bundle with zero section $F, f_{1}$ is a covering of $F$ onto $G$, one component of $N-G$ bas closure $G \times[0, \infty)$ and $f_{1}(k, t)=\left(f_{1}(k),|t|\right)$ where $|t|$ is with respect to some Riemannian metric on $M$. Furthermore, if $f \mid \partial M$ is a local homeomorphism, then the bomotopy may be chosen constant on $\partial M$.

Lemma 4.2. Let $M$ be an irreducible, end-irreducible manifold, and $G$ an incompressible closed surface in $M$. Let $H: M \times I \rightarrow M$ be a bomotopy of a bomeomorphism $b$ to the identity. Suppose $b(G)=G$ and $H_{G}(G \times I) \subset G$. If $M$ is bomeomorphic to $G \times R$, where $R$ is the real line, assume $b$ is orientation preserving. Then $b$ does not interchange the sides of $G$. 
Proof. If $M-G$ is connected the proof is the same as for (2.19).

If $M-G$ is disconnected, let $A$ and $B$ denote the closures of the components of $M-G$. Then $\pi_{1}(M)=\pi_{1}(A) *_{\pi_{1}(G)} \pi_{1}(B)$. If this is nontrivial, i.e., $\pi_{1}(G) \rightarrow \pi_{1}(B)$ is not surjective, and our conclusion is assumed false, then the argument Waldhausen gives in [13, Lemma 7.4, p. 83] shows this leads to a contradiction.

If our representation of $\pi_{1}(M)$ is trivial and we assume that $b(A)=B$ then $\pi_{1}(G) \rightarrow \pi_{1}(A)$ is also an isomorphism. We will show that this implies $M=G \times R$.

We claim that $A$ is homeomorphic to $G \times[0, \infty)$. The inclusion map $i: G \rightarrow A$ induces a homotopy equivalence between $G$ and $A$. Let $i^{-1}: A \rightarrow G$ be a homotopy inverse. Let $f: A \rightarrow[0, \infty)$ be a proper map so that $f(G)=0$. Then the map $i^{-1} \times f: A \rightarrow G \times[0, \infty)$ is proper. Moreover $\left(i^{-1} \times f\right)_{*}^{-1}$ is an isomorphism. Thus we apply (4.1) to conclude that $i^{-1} \times f$ is homotopic to a homeomorphism. Hence $A=G \times[0, \infty)$. Thus we get $M=G \times R$, and since $b$ is orientation preserving on all of $M$, it cannot interchange $A$ and $B$.

We have two isotopy results for end-irreducible manifolds. They correspond to (3.6) and (3.7); depending on whether or not $H(\partial M \times I)$ is contained in $\partial M$. Precisely, we show

Theorem 4.3. Let $M$ be a noncompact irreducible, end-irreducible manifold. Let $H:(M \times I, \partial M \times I) \rightarrow(M, \partial M)$ be a bomotopy of pairs of a bomeomorphism $b$ to the identity. Assume that if $F$ is a boundary component of $M$ which is a plane or an open annulus $b \mid F$ is orientation preserving. Assume further that $b$ is orientation preserving if $M$ is a bundle over a closed surface (possibly nonorientable) with fiber $R$. Then $b$ is isotopic to the identity. If $H \mid \partial M \times I$ is projection onto $\partial M$, the isotopy of $b$ to the identity may be chosen constant on $\partial M$.

Theorem 4.4. Let $M$ be an irreducible, end-irreducible manifold with $\partial M \neq \varnothing$. Suppose $M$ is boundary-irreducible and has no boundary components which are planes. Let $H: M \times I \rightarrow M$ be a bomotopy of a bomeomorphism $b$ to the identity such that for each boundary component $F, H \mid F \times I$ is a proper map. If $M$ is a bundle over a noncompact surface (possibly nonorientable) with fiber I, assume $b$ is orientation preserving. Then $b$ is isotopic to the identity.

The assumption that $H \mid F \times I$ is proper is essential; for let $M$ be a solid torus with two disjoint longitudinal curves removed from its boundary. $\partial M$ consists of two open annuli. The rotation of the torus which takes one annulus onto the next is homotopic to the identity. Clearly since it interchanges boundary components it cannot be isotopic to the identity.

Proof of Theorem 4.3 when all boundary components are compact. The first step is exactly the same as Step 1 of Theorem 3.6. We can go on assuming $b$ and $H$ are fixed up on $\partial M ; b$ has been isotoped to a homeomorphism which is the 
identity on $M$ and $H$ has been changed so that when restricted to $\partial M$ it is projection onto the first factor.

Choose an exhausting sequence $\left\{C_{i}\right\}$ for $M$ satisfying (3.1). In addition, choose $\left\{C_{i}\right\}$ so that $H\left(C_{i} \times I\right)$ is contained in $C_{i+1}$. We will define our isotopy of $b$ to the identity by an inductive procedure. That is, we will isotope $b$ to the identity on $C_{i-1}$ rel $\partial M$. Then we will construct an isotopy which is fixed on $C_{i-1}$ and $\partial M$ and changes $b \mid C_{i}$ to the identity. Hence all these put together give a well defined isotopy of $b$ to the identity.

Let us assume $b \mid U\left(C_{i-1}\right)$ is the identity and $H \mid U\left(C_{i-1}\right) \times I$ is projection onto the first factor. We now fix $b$ and $H$ on the shell cl $\left(U\left(C_{i}\right)-U\left(C_{i-1}\right)\right)$. Let $C_{0}=\varnothing$.

(1) Let $F_{1}, \ldots, F_{k}$ be the components of $\operatorname{Fr} C_{i}$. By (3.1), each $F_{i}$ is closed and incompressible in $M$ and hence certainly in $C_{i+1}$. We apply (1.1) with $C=$ $C_{i+1}$, the system $F=\operatorname{Fr} U\left(C_{i-1}\right)$ and $G=F_{1}$. (Use (4.2) to see that $b$ does not interchange the sides of the components of $F$.) We obtain an isotopy of $C_{i+1}$, which is fixed on $\partial C_{i+1} \cup \operatorname{Fr} U\left(C_{i-1}\right)$ carrying $h\left(F_{1}\right)$ onto $F_{1}$. Since $F_{r} U\left(C_{i-1}\right)$ separates $C_{i+1}$, and one of the components of the complement is $U\left(C_{i-1}\right)$ and the isotopy is fixed on $\partial U\left(C_{i-1}\right)=\operatorname{Fr} U\left(C_{i-1}\right)$, we actually have an isotopy on $\mathrm{cl}\left(C_{i+1}-U\left(C_{i-1}\right)\right)$ rel the boundary which takes $b\left(F_{1}\right)$ onto $F_{1}$. We extend this isotopy trivially to all of M. Changing $b$ and $H$ by this isotopy we continue under the assumption that $b\left(F_{1}\right)=F_{1}$.

(2) We would like to homotope $H_{F_{1}}: F_{1} \times I \rightarrow C_{i+1}$ rel $F_{1} \times \partial I$ to a map into $F_{1}$.

Case 1. $C_{i}$ bas more boundary than $F_{1}$. We apply (2.8) to get our result; for if $\partial C \neq F_{1}$, then if $F_{1}$ separates $C_{i+1}$ it also separates $\partial C_{i+1}$.

Case 2. $\partial C_{i}$ is connected. Discard it from our exhausting sequence. If one can find a subsequence of $\left\{C_{i}\right\}$ so that each member has nonconnected boundary we apply (2.8) to homotope $H_{F_{1}}$ and continue with the proof. If $\partial C_{i}$ is connected for each $i$, but still we can find a homotopy of $H \mid \partial C_{i} \times I$ rel $\partial C_{i} \times \partial I$ to a map into $\partial C_{i}$, we again have that (2) is true and we go on. We consider at the end of this proof the case in which $\partial C_{i}$ is connected for each $i$ and there is no homotopy of $H \mid \partial C_{i} \times I$ to a map into $\partial C_{i}$. Thus except for the last mentioned case we now have a homotopy of $H_{F_{1}}$ rel $F_{1} \times I$ to a map into $F_{1}$.

The rest of the proof is now quite similar to the proof of Theorem (3.6). This proves (4.3) except for the case in which $\partial C_{i}$ is connected for each $i$ and $H \mid \partial C_{i}$ $\times I$ is not homotopic rel $\partial C_{i} \times I$ to a map into $\partial C_{i}$. This can occur only when $\partial M=\varnothing$. In fact this happens only when $M$ is a bundle over a nonorientable closed surface with fiber $R$ and $b$ is orientation-reversing. That is, this cannot occur under the hypotheses.

We first show that $C_{i}$ is homeomorphic to a bundle over a nonorientable closed 
surface with fiber $I$. Since $H\left(\partial C_{i} \times I\right)$ is contained in $C_{i+1}$, and both $\partial C_{i}$ and $b\left(\partial C_{i}\right)$ are incompressible in $C_{i+1}$, by (3.2) there is an isotopy of $M$ fixed outside of $C_{i+1}$ carrying $b\left(\partial C_{i}\right)$ onto $\partial C_{i}$. Change $b$ and $H$ according to this isotopy so that now $b\left(\partial C_{i}\right)=\partial C_{i}$. Let $F$ be a homeomorphic copy of $\partial C_{i}$, and define the map $H^{\prime}: F \times I \rightarrow C_{i+1}$ as the restriction $H \mid \partial C_{i} \times I$. Given a regular neighborhood of $F \times \partial I$ in $F \times I$ there is a homotopy of $H^{\prime}$ rel $F \times \partial I$ so that afterwards $H^{\prime-1}\left(\partial C_{i} \cap U(F \times \partial I)\right)=F \times \partial I$. Follow (2.8) exactly up to Case 1 . Summarizing, we have homotoped $H^{\prime}$ rel $F \times \partial I$ so that $H^{\prime-1}\left(\partial C_{i}\right)=F \times \partial I \cup$ $F_{1} \cup \cdots \cup F_{m}$, where each $F_{i}$ is an incompressible surface in $F \times I$ which is parallel to $F \times 0$. In addition, at this point assume $H^{\prime}$ has been homotoped so that the number of $F_{i}$ 's is minimal. Let us denote by $\widetilde{C_{i+1}}$ the result of splitting $C_{i+1}$ along $\partial C_{i}$. Lift $H^{\prime} \mid F \times I^{\prime}$ to $\widetilde{C_{i+1}}$. We continue independently of (2.8) now. We apply [13, Theorem 6.1] to each of the $\overline{H^{\prime} \mid F \times I^{\prime}}$. If each one was homotopic rel $F \times \partial I^{\prime}$ to a map into $\widehat{\partial C_{i+1}}$ this would imply $H^{\prime}: F \times I \rightarrow C_{i+1}$ was homotopic to a map into $\partial C_{i}$. We assumed at the start that this did not occur. Hence some $\overline{H^{\prime} \mid F \times I^{\prime}}$ is homotopic to a covering map. (In fact, since the number of $F_{i}$ 's is assumed minimal, we have that all of the $H^{\prime} \mid F \times I^{\prime \prime}$ s are homotopic to coverings). The covering maps go onto the component of $\widetilde{C_{i+1}}$ homeomorphic to $C_{i}$. Since $H^{\prime} \mid F \times I^{\prime}$ is a homeomorphism on each component of $F \times \partial I^{\prime}$, it is homotopic to a $2-1$ covering. By $[13, \mathrm{p} .70]$ we can conclude that $C_{i}$ is a bundle with fiber $I$ over a nonorientable closed surface.

Later in the proof we will need that all of $H^{\prime} \mid F \times I$ is homotopic rel $F \times \partial I$ to a 2-1 covering. It suffices to show that if $m$ is the minimum number of $F_{i}$ 's then $m=0$. If this were not so, two adjacent liftings of $H^{\prime} \mid F \times I^{\prime}$ are homotopic to coverings. Denote them by $H_{1}: F \times I_{1} \rightarrow C_{i}$ and $H_{2}: F \times I_{2} \rightarrow C_{i}$. Then $H_{1}$ followed by $H_{2}$ is homotopic to a map into $\partial C_{i}$. This contradicts our minimality assumption.

Next we show that $M$ is homeomorphic to a bundle with fiber $R$ over a nonorientable closed surface. In fact if $B_{i}$ is the bundle with fiber $R$ associated with the bundle $C_{i}$, then $M$ is homeomorphic to $B_{i}$ by a homeomorphism which "extends" $H: \partial C_{i} \times I \rightarrow C_{i}$.

Let $\lambda$ be a loop on $\partial C_{i}$, and let $\rho$ be a path in $\mathrm{cl}\left(C_{i+1}-C_{i}\right)$ from $\partial C_{i+1}$ to the base point of $\lambda$. Then twice around $\left(\rho \lambda \rho^{-1}\right)$ is homotopic to a loop in $\partial C_{i+1}$ since $\pi_{1}\left(\partial C_{i+1}\right)$ has index 2 in $\pi_{1}\left(C_{i+1}\right)$. But using the fact that $\partial C_{i}$ is incompressible in $C_{i+1}$ and Van Kampen's theorem one can show that $\pi_{1}\left(\mathrm{cl}\left(C_{i+1}-C_{i}\right)\right) \rightarrow \pi_{1}\left(C_{i+1}\right)$ is a monomorphism. Therefore $\lambda$ is homotopic in $\mathrm{cl}\left(C_{i+1}-C_{i}\right)$ to a loop in $\partial C_{i+1}$. By $\left[13\right.$, Lemma 5.1] $\mathrm{cl}\left(C_{i+1}-C_{i}\right)$ is homeomorphic to $\partial C_{i} \times I$. Similarly for each shell. Hence $M$ is homeomorphic to an $R$-bundle over the same nonorientable closed surfaces as $C_{i}$. The argument given 
on p. 83 of [13] shows that if $b$ is orientation preserving, we reach a contradiction.

Proof of (4.3) when some component of $\partial M$ is noncompact. $b \mid \partial M$ is isotopic to the identity. Proceeding as in the previous theorem of this section we may assume $b \mid \partial M$ is the identity and that $H$ is projection onto $\partial M$.

Now that $H$ is fixed up on the boundary, the only difference remaining in the noncompact case is that if $\left\{C_{n}\right\}$ is an exhausting sequence satisfying (3.1), $\operatorname{Fr} C_{n}$ may contain components which are not closed. (2.11) takes care of such surfaces.

Proof of Theorem 4.4 when all components of $\partial M$ are compact. Using [13, Theorem 6.1] one can reduce this to (4.3). For the proof of (4.4) when we allow noncompact boundary components we need a noncompact version of [13, Proposition 4.1].

The twisted-bundle result. We will say $f:(M, \partial M) \rightarrow(N, \partial N)$ contracts as a map of pairs if $f$ is homotopic by a homotopy of pairs to a map $g:(M, \partial M) \rightarrow$ $(\partial N, \partial N)$. If $t$ is a homeomorphism of $M$ onto itself we say $N \subset M$ is $t$-invariant or invariant under $t$ if $t(N)=N$.

Proposition 4.5. Let $F$ be a noncompact surface without boundary, different from a plane. Let $M$ be a manifold with connected boundary. Suppose $f: F \times I \rightarrow$ $M$ is a 2-1 covering map which is proper. Then $M$ is a bundle with fiber I over a nonorientable noncompact surface.

Proof. From the hypotheses of the theorem it is easy to see that $f \mid F \times 0$ and $f \mid F \times 1$ are homeomorphisms onto the boundary of $M$. Thus we can regard $F$ as the boundary of $M$ and identify points of $F$ so that $(x, 0)$ corresponds to $x$ in $F$ via $f \mid F \times 0$. We denote $f \mid F \times i, i=0$ and 1 , by $f_{i}$ and regard them as homeomorphisms of $F$ onto itself. $f_{0}$ is the identity function. At the beginning of Step 1 we refer to $f_{1}$. The above function is the one we mean.

Let $t: F \times I \rightarrow F \times I$ denote the covering transformation. We will construct a fibering of $F \times I$ which is invariant under $t$ in order to prove the result. We handle the case where $F$ is an open cylinder at the end.

The bulk of the proof consists in producing an exhausting sequence for $F \times I$, which is invariant under $t$ and whose frontier components consist of annuli which are pairwise interchanged under $t$. After that, the proof uses the techniques of the compact version in [13].

Step 1 . We begin by constructing an exhausting sequence $\left\{G_{n}\right\}$ for $F$ which consists of compact connected surfaces which are invariant under $f_{1}$ and such that $F-G_{n}$ has only unbounded components. Let $\left\{F_{n}\right\}$ be an exhausting sequence for $F$ consisting of compact connected surfaces. Since $F$ is not an open annulus assume $F_{1}$ is not an annulus or disc. Small deformations of the $F_{i}$ 's will insure that $\partial F_{i}$ and $f_{1}\left(\partial\left(F_{i}\right)\right)$ intersect transversally. Hence $F_{i} \cup f_{1}\left(F_{i}\right)$ is a surface. Moreover, if $n$ is sufficiently large, $F_{n} \cup f_{1}\left(F_{n}\right)$ is connected. 
By eliminating the first few $F_{i}$ 's, $\left\{F_{i} \cup f_{1} F_{i}\right\}$ is an exhausting sequence of connected surfaces which are invariant under $f_{1}$. If for some $n, F-\left(F_{n} \cup f_{1}\left(F_{n}\right)\right)$ has some components with compact closure we add them to $F_{n} \cup f_{1}\left(F_{n}\right)$. Since there are only finitely many boundary components of $F_{n} \cup f_{1} F_{n}$ this set is still compact. Let us denote the resulting exhausting sequence by $\left\{G_{n}\right\}$.

Step 2 . We now modify $G_{1} \times I$ so that the resulting set which we will denote by $C_{1}$ is a $t$-invariant set whose frontier components are annuli which are interchanged by $t . C_{1}$ will be the first member of the exhausting sequence we want.

(1) Let $\lambda$ be a boundary loop of $G_{1}$. We can deform $\lambda \times I$ so that afterwards $\lambda \times I \cap t(\lambda \times I)$ consists only of simple closed double curves and double arcs.

(2) Denote $\lambda \times I$ by $A_{\lambda}$. Intersections of the frontiers occur only when one annulus is an $A_{\lambda}$ and one is a $t A_{\mu}$. We wish to alter the $A_{\lambda}$ so that $t\left(A_{\lambda}\right)=A_{\mu}$. We first modify the $A_{\lambda}$ so that whenever $A_{\lambda} \cap t A_{\mu}$ is nonvacuous $A_{\lambda} \cap t A_{\mu}=A_{\lambda}$ or $\partial A_{\lambda}$.

It is not difficult to first change $G_{1} \times I \cup t G_{1} \times I$ so that the intersections of the resulting annuli are only noncontractible intersection loops or only arcs which intersect both $F \times 0$ and $F \times 1$. Clearly the intersection arcs occur only for pairs $A_{\lambda}, t A_{f_{1}^{-1}(\lambda)}$ for some $\lambda$ in $G_{1}$. The same is true of the intersection loops since $F$ is not an open annulus.

(a) $A_{\lambda} \cap t A_{\mu}$ consists of simple closed curves which are parallel to $\lambda \times 0$. (a-1) $\lambda=\mu$. Take a small regular neighborhood $U\left(A_{\lambda} \cup t\left(A_{\lambda}\right)\right)$ which is invariant under $t$. $\operatorname{Fr}\left(U\left(A_{\lambda} \cup t A_{\lambda}\right)\right)$ contains two incompressible annuli $A^{\prime}$ and $A$ " which intersect both $F \times 0$ and $F \times 1$. Each frontier component is either a torus or an annulus. If it is an annulus with both boundary loops on $F \times 0$ say, then it would disconnect $\lambda \times 0$ from $\lambda \times 1$. This would be possible only when $F$ is a torus. But $F$ is noncompact. Therefore our assertion about $A^{\prime}$ and $A^{\prime \prime}$ is true. If $t\left(A^{\prime}\right)=A^{\prime}$, then $f\left(A^{\prime}\right)$ ( $f$ is the covering map) would be a 2-sided Moebius band in $M$. Since $M$ is orientable this cannot occur. On the other hand if $t\left(A^{\prime}\right)=$ $A^{\prime \prime}$, this would contradict the fact that $G_{1}$ is invariant under $f_{1}$. Thus we have shown that $(\mathrm{a}-1)$ cannot occur; i.e., $\lambda=\mu$. Note that this same argument also shows that $t\left(A_{\lambda}\right) \neq A_{\lambda}$. We shall keep this in mind for a later argument.

$(\mathrm{a}-2) \lambda \neq \mu$. In this case since we have shown that at most one pair of annuli intersect; $A_{\lambda} \cup t A_{\mu}$ and $t A_{\lambda} \cup A_{\mu}$ are disjoint sets. Let $U\left(A_{\lambda} \cup t A_{\mu}\right)$ be a regular neighborhood of $A_{\lambda} \cup t A_{\mu}$ so that $t\left(U\left(A_{\lambda} \cup t A_{\mu}\right)\right) \cap U\left(A_{\lambda} \cup t A_{\mu}\right)=\varnothing$. As in (a-1), Fr $U\left(A_{\lambda} \cup t A_{\mu}\right)$ contains two incompressible annuli $A^{\prime}$ and $A^{\prime \prime}$ each one intersecting both $F \times 0$ and $F \times 1$. Since $t\left(U\left(A_{\lambda} \cup t A_{\mu}\right)\right) \cap U\left(A_{\lambda} \cup t A_{\mu}\right)=\varnothing$, $t A^{\prime} \cap A^{\prime}=t A^{\prime \prime} \cap A^{\prime \prime}=\varnothing$. Replace $A$ with one of the $A^{\prime \prime}$ 's modified slightly so that $\partial A^{\prime}=\lambda \times 0 \cup \lambda \times 1$.

(b) $A_{\lambda} \cap t A_{\mu}$ consists only of arcs, each of which intersects both $F \times 0$ and 
$F \times 1$. First of all it is not possible that $A_{\lambda} \cap t A_{\mu}$ consists of just one arc $\rho$; for since $A_{\lambda}$ intersects $t A_{\mu}$ transversally along int $\rho$ this would imply that $\lambda$ has intersection number 1 with itself. But $F$ is orientable, so this is impossible. Therefore two consecutive arcs $\rho_{1}, \rho_{2}$ on $A_{\lambda}$ cut off discs $D$ and $D^{\prime}$ on $A_{\lambda}$ and $t A_{\mu}$ respectively. It is not hard now to eliminate at least $\rho_{1}$ and $\rho_{2}$.

Thus we have modified the frontier components $A_{\lambda}$ of $G_{1} \times I$ so that for each boundary loop $\lambda, t A_{\lambda}=A_{\mu}$ and $\lambda \neq \mu$. We call this modified set $C_{1}$. It qualifies as our first term of an exhausting sequence $\left\{C_{i}\right\}$ for $F \times I$ so that each $C_{i}$ is a connected manifold which is invariant under $t$ and whose boundary components are annuli which are interchanged by $t$.

By $[13$, p. 68] there is an isotopy of $F \times I$ constant on $F \times \partial I$ which makes all the components of $\operatorname{Fr}_{r} C_{1}$ vertical. Instead let us use the inverse of the isotopy. to change the product structure on $F \times I$.

Step 3. We now provide an inductive procedure for constructing the $C_{i}$ 's. Having constructed $C_{i}$, first choose $m$ sufficiently large so that $\left[\mathrm{Fr}_{\mathrm{r}}\left(G_{m} \times I\right) \cup\right.$ $\left.\mathrm{Fr}_{\mathrm{r}}\left(t\left(G_{m} \times I\right)\right)\right] \cap\left(C_{i} \cup G_{i+1} \times I\right)=\varnothing$. Now change $G_{m} \times I \cup t\left(G_{m} \times I\right)$ as we changed $G_{1} \times I \cup t\left(G_{1} \times I\right)$ to construct $C_{i+1}$. We use $[13, \mathrm{p} .68]$ to "straighten up" $\operatorname{Fr} C_{i+1}$ in $\mathrm{cl}\left(F \times I-C_{i}\right)$. In fact, we can regard the isotopies as taking place in shells of the form $\mathrm{cl}\left(G_{n(i+1)}-G_{n(i)}\right) \times I$, which contain the frontier of only one of the $C_{i}$ 's. Thus we can combine them to get a global isotopy of $F \times I$ so that for each $n$ Fr $C_{n}$ is vertical. Moreover, $\left\{C_{i}\right\}$ exhausts $F \times I$, since at each stage of the construction we chose $C_{i}$ so that it contained our original $G_{i} \times I$.

Step 4. We begin to construct the fibering of $F \times I$ which is invariant under $t$. We first do it on regular neighborhoods of the frontiers of $C_{n}$.

Fix $n$. Let $A$ be a component of $\operatorname{Fr} C_{n}$. We make $t \mid A$ fiber preserving by deforming $M \times I$ near $t(A)$ by an isotopy which takes $t(A)$ 's "natural" fibering to the one which is defined by $t: A \rightarrow t A$. Using this isotopy we can get vertical neighborhoods $U(A)$ and $U(t A)$ such that $t(U(A))=u(t A)$ and such that $t \mid U(A)$ is fiber preserving.

Step 5. We make $t$ fiber preserving on the rest of $F \times I$, exactly as Waldhausen suggests in [13, Proposition 4.1].

We are now finished except when $F$ is an open annulus. A similar but much simplified proof will work.

An outline of the proof of (4.4) when some component is not compact. If $H \mid \partial M \times I \rightarrow M$ is homotopic rel $\partial M \times \partial I$ to a map into $\partial M$, then by (4.3) the result follows. If however there exists a noncompact component $F$ of $\partial M$ such that $H \mid F \times I$ is not homotopic to a map into $F$, then we show that either

(a) $H \mid F \times I$ is homotopic to a homeomorphism of $F \times I$ onto $M$, or

(b) $H \mid F \times I$ is homotopic to a $2-1$ covering of $F \times I$ onto $M$. 
Case (a) occurs if we assume $b(F) \neq F$, while (b) occurs if $b(F)=F$. In either case we get a contradiction.

Proof. We begin by assuming there is a noncompact component $F$ for which $H: F \times I \rightarrow M$ is not homotopic to a map into $F$.

Part 1. We show $H$ is homotopic to a covering of $F \times I$ onto $M$. Essentially we are mimicking the proof of [13, Theorem 6.1].

Choose an exhausting sequence $\left\{C_{n}\right\}$ for $M$ satisfying (3.1).

(1) There is a proper homotopy of $H$ to a map which we continue to denote by $H$ so that $H^{-1}\left(C_{n}\right)$ is a compact manifold with incompressible frontier components. One can do this using (2.13) and the fact that $H$ is proper.

Let $\left\{D_{n}\right\}$ be an exhausting sequence for $F \times I$ with each $\left\{D_{n}\right\}$ a component of $H^{-1}\left(C_{n}\right)$.

(2) We show that for every $n$, and every component $G$ of $\operatorname{Fr} D_{n}, H \mid G: \rightarrow$ Fr $C_{n}$ is homotopic rel $\partial G$ to a covering. For if we argue as in $[13, \mathrm{p} .78]$ one can conclude that $H$ is homotopic to a map into $F$ rel $F \times \partial l$. We are assuming $H \mid F \times I$ is not homotopic to a map into $F$; so (2) follows. Thus $H$ is proper homotopic to a map which is a covering when restricted to $\operatorname{Fr} D_{n}$ fcr each $n$. Furthermore since $H\left(D_{n} \cap(F \times I)\right) \cap H\left(\right.$ int $\left.F_{r} D_{n}\right)=\varnothing, H \mid \partial D_{n}$ is a local homeomorphism.

(3) Next we prove $H: F \times I \rightarrow M$ is proper homotopic rel $F \times \partial I$ to a covering.

Choose hierarchies for $S_{0}=C_{1}, S_{n}=\mathrm{cl}\left(C_{n+1}-C_{n}\right), n=1,2, \cdots$; let $S_{n}^{1}=S_{n}, F_{j} \subset S_{n}^{j}, U\left(F_{j}\right) \subset S_{n}^{j}, S_{n}^{j+1}=\mathrm{cl}\left(S_{n}^{j}-U\left(F_{j}\right)\right), j=1, \cdots, k_{n}$.

(a) Fix $n$; we will show that $H \mid H^{-1}\left(\partial C_{n} \cup \bigcup_{j=1}^{k_{j}} U\left(F_{j}\right)\right) \cap D_{n} \rightarrow S_{n}$ is homotopic to a local homeomorphism. By the above it is true for $j=0$. Suppose we have proved it for all $j<l$. By $[13$, p. 60] there is a homotopy of $H$ : $\operatorname{cl}\left(D_{n+1}-D_{n}\right) \rightarrow S_{n}=\operatorname{cl}\left(C_{n+1}-C_{n}\right)$ rel $\partial\left(\operatorname{cl}\left(D_{n+1}-D_{n}\right)\right)$ so that afterwards $H$ is transverse with respect to $F_{l}$ and so that $H^{-1}\left(F_{l}\right)$ is a system of incompressible surfaces in $\mathrm{cl}\left(D_{n+1}-D_{n}\right)$. Then $\operatorname{ker}\left(H_{*}\right)=0$; it follows that if $G$ is any component of $H^{-1}(F)$ either $H \mid G: \rightarrow F_{l}$ is homotopic to a covering - in which case (3) follows - or we have one of two things occurring:

(i) $G$ is a disc and hence so is $F_{l}$ and the covering map $H \mid \partial G$ is not a homeomorphism, or

(ii) by $\left[13\right.$, Lemma 1.4.3], $G$ is an annulus and $H \mid G:(G, \partial G) \rightarrow\left(F_{l}, \partial F_{l}\right)$ contracts to $\left(\partial F_{l}, \partial F_{l}\right)$. Note that here $H(\partial G)$ is contained in one boundary curve of $F_{l}$

Both (i) and (ii) above allow us to choose a simple arc $\rho$ which is properly embedded in $F \times I$ so that $H(\partial \rho)$ is one point and $H \mid \rho:(\rho, \partial \rho) \rightarrow(F, H(\partial \rho))$ contracts. This implies that $H \mid F \times I \rightarrow M$ is homotopic to a map into $F$. We have assumed this is not so. Thus we conclude that $H$ is proper homotopic 
rel $\partial(F \times I)$ to a map which is a covering on all but a collection of 3-cells of $F \times I$. Hence for each 3 -cell $C, H$ restricted to $C$ is homotopic rel $\partial C$ to a homeomorphism. Since $H$ is proper and $M$ is not $R^{3}, H$ is actually proper homotopic to a covering on all of $F \times I$; i.e., $H$ is surjective.

Thus we conclude the proof of Part 1 , that is, if $F$ is not a plane, and $H$ : $F \times I \rightarrow M$ does not contract then $H$ is homotopic rel $F \times \partial I$ to a homeomorphism or a $2-1$ covering of $F \times I$ onto $M$.

Part 2. We show that the conclusion of Part 1 leads us to a contradiction.

(a) Suppose $H$ is $1-1$, then $M=F \times I$. In this case we are assuming $b$ is orientation-preserving. Using $H: F \times I \rightarrow M$, identify $F$ with $F \times 0$. Then $b(F \times 0)=F \times 1 . \quad b \mid F \times 0$ followed by projection of $M$ onto $F \times 0$ is a homeomorphism $b^{\prime}$ of $F \times 0$ onto itself which is proper homotopic to the identity. By (3.3) $b^{\prime}: F \times 0 \rightarrow F \times 0$ is isotopic to the identity. Therefore $b^{\prime}: F \times 0 \rightarrow$ $F \times 0$ is orientation-preserving; but then so is $b: F \times 0 \rightarrow F \times 1$. Since $b$ interchanges $F \times 0$ and $F \times 1, b: M \rightarrow M$ is orientation-reversing.

(b) If $H$ is $2-1$, by (4.5) $M$ is a bundle with fiber $I$; hence we have $b$ is orientation-preserving. See $[13$, p. 83$]$ to see that leads to a contradiction.

\section{REFERENCES}

1. R. Baer, Kurventypen auf Flächen, J. Reine Angew. Math. 156 (1927), 231-246.

2. - Isotopie von Kurven auf orientbaren, geschossenen Flächen und ihr Ausammenhang mit der topologischen Deformation der Flächen, J. Reine Angew. Math. 159 (1928), 101-116.

3. R. Bing, Locally tame sets are tame, Ann. of Math. (2) 59 (1954), 145-158. MR 15, 816 .

4. E. M. Brown, On proper homotopy type, Proc. Topology Conf., Virginia Polytechnic Institute, Blacksburg, Va. (to a ppear).

5. E. M. Brown and T. Tucker, On proper homotopy theory for noncompact 3-manifolds, Trans. Amer. Math. Soc. (to a ppear).

6. D. B. A. Epstein, Curves on 2-manifolds and isotopies, Acta Math. 115 (1966), 83-107. MR 35 \#4938.

7. C. D. Feustel, Isotopic unknotting in $M^{2} \times I$, Thes is, Dartmouth College, Hanover, N. H.

8. G. Fisher, On the group of all homeomorphisms of a manifold, Trans. Amer. Math. Soc. 97 (1960), 193-213. MR 22 \#8487.

9. W. Haken, Über das Homöomorphieproblem der 3-Mannigfaltigkeiten. I, Math. Z. 80 (1962), 89-120. MR 28 \#3410.

10. J. Kister, Isotopies in 3-manifolds, Trans. Amer. Math. Soc. 97 (1960), 213-224. MR $22 \# 11378$.

11. D. R. McMillan, Jr., Some contractible open 3-manifolds, Trans. Amer. Math. Soc. 102 (1962), 373-382. MR $25 \# 561$.

12. E. E. Moise, Affine structures in 3-manifolds. VIII. Invariance of the knot-types, local tame imbedding, Ann. of Math. (2) 59 (1954), 159-170. MR 15, 889. 
13. F. Waldhausen, On irreducible 3-manifolds which are sufficiently large, Ann. of Math. (2) 87 (1968), 56-88. MR 36 \#7146.

14. J. H. C. Whitehead, A certain open manifold whose group is unity, Quart. J. Math. Oxford 6 (1935), 268-279.

15. - Simplicial spaces, nuclei, and m-groups, Proc. London Math. Soc. 45 (1937), 243-327.

16. J. H. C. Whitehead and A. Shapiro, A proof and extension of Dehn's lemma, Bull. Amer. Math. Soc. 64 (1958), 174-178.

17. J. H. C. Whitehead and M. H. A. Newman, On the group of a certain linkage, Math. Works of J. H. C. Whitehead., vol. II, Macmillan, New York, 1963.

18. M. S. Brown, Constructing isotopies in noncompact 3-manifolds, Bull. Amer. Math. Soc. 78 (1972), 461-464.

DEPARTMENT OF MATHEMATICS, DARTMOUTH COLLEGE, HANOVER, NEW HAMPSHIRE 03755 